\title{
Familia Ellobiidae (Gastropoda: Archaeopulmonata) en el litoral peruano
}

\section{Family Ellobiidae (Gastropoda: Archaeopulmonata) in the Peruvian coast}

\author{
Carlos Paredes ${ }^{1}$, Aldo Indacochea ${ }^{2}$, Franz Cardoso $^{1}$ y Kelly Ortega ${ }^{2}$ \\ Presentado: $12 / 07 / 2005$ \\ Aceptado: $\quad 01 / 08 / 2005$
}

\section{Resumen}

Se reportan 8 especies de Ellobiidae para la Costa Peruana, pertenecientes a las subfamilias Ellobiinae: Ellobium stagnale (Orbigny, 1835) y Sarnia frumentum Petit, 1842; Melampodinae: Melampus carolianus (Lesson, 1842), Melampus olivaceus Carpenter, 1857 y Detracia graminea Morrison, 1846; y Pedipedinae: Marinula acuta (Orbigny, 1835), Marinula concinna (C.B Adams, 1852) y Marinula pepita King, 1831. Seis especies viven asociadas al bosque de manglar en el departamento de Tumbes, y dos en las playas de canto rodado en los límites de la Provincia Peruana. Cuatro especies tropicales se registran por primera vez para el mar peruano: E. stagnale, M. olivaceus, D. graminea y $M$. acuta.

Palabras clave: Mollusca, Ellobiidae, taxonomía, nuevos reportes, Perú.

\section{Abstract}

We report 8 species of Ellobiidae from the Peruvian coast, which belong to the subfamilies Ellobiinae: Ellobium stagnale (Orbigny, 1835) and Sarnia frumentum Petit, 1842; Melampodinae: Melampus carolianus (Lesson, 1842), Melampus olivaceus Carpenter, 18574 and Detracia graminea Morrison, 1846; and Pedipedinae: Marinula acuta (Orbigny, 1835), Marinula concinna (C.B Adams, 1852) and Marinula pepita King, 1831. Six species live associated to the mangrove of the department of Tumbes, and two in boulder beaches in the limits of the Peruvian Province. Four tropical species are registered for the first time in the Peruvian Sea: A. stagnale, M. olivaceus, D. graminea and M. acuta.

Keywords: Mollusca, Ellobiidae, taxonomy, new records, Peru.

\section{Introducción}

Los moluscos gasterópodos conocidos como «pulmonados marinos de concha cónica» (Hyman, 1967) pertenecientes a la familia Ellobiidae, que viven en el Perú, son casi desconocidos no obstante su importancia biológica y ecológica. Los Ellobiidae constituyen un grupo primitivo de moluscos pulmonados, mayormente marinos, habitantes de los niveles superiores de la zona intermareal, en diversos tipos de hábitat como los manglares, las playas pedregosa y de canto rodado.

Para el mundo, Goto y Poppe (1996), listan 154 especies nominales de Ellobiidae distribuidas en 21 géneros; Vaught (1989) también considera el mismo número de géneros, pero

\footnotetext{
${ }^{1}$ Laboratorio de Biología y Sistemática de Invertebrados Marinos, Facultad de Ciencias Biológicas, UNMSM, Apdo. 11-0058, Lima 11, Perú y Departamento de Malacología, Museo de Historia Natural, UNMSM, Apdo. 14-0034, Lima 14, Perú. E-mail Carlos Paredes: cparedesq@unmsm.edu.pe

${ }^{2}$ Laboratorio de Ecología Marina, Facultad de Ciencias Biológicas, UNMSM.
}

Millard (1997) eleva este número a 26. Para el Pacífico Este Tropical, Goto y Poppe (1996) señalan 69 especies nominales incluidas en 7 géneros; Keen (1971) registra 6 géneros y 20 especies. Cruz y Jiménez (1994) hallaron 7 especies asociadas al manglar en Colombia. En la actualización de Paredes et al. (1999), se listan 2 especies para el mar tropical del Perú y 3 en la Provincia Peruana; éstas últimas, también son conocidas en Chile (Carcelles y Williamson, 1951; Marincovich, 1973; Letelier et al., 2003).

En cuanto a la sistemática de este grupo, los Ellobiidae incluyen 3 subfamilias: Ellobiinae, Melampodinae y Pedipedinae(Keen, 1971; Vaugh, 1989; Millard, 1997), división basada en criterios conquiológicos. Sin embargo, Martins (1996) estudiando la anatomía del sistema nervioso y el sistema reproductor, especialmente el complejo peneano, considera a la familia Carychidae como la cuarta subfamilia de Ellobiidae.

El objetivo de este trabajo ha sido contribuir al conocimiento de la diversidad de los 
Ellobiidae, actualizar la taxonomía de la familia, y realizar observaciones biológicas, dada su importancia en la ecología de los manglares y las playas de la costa peruana.

\section{Material y métodos}

Se ha estudiado el material colectado durante más de 25 años, en diversos lugares y tipos de playa de la Costa Peruana. Las colectas se realizaron en localidades como Tumbes (El Bendito, Estero de Zarumilla, Malpelo, Isla del amor y Puerto Pizarro), Lima (La Punta, Las Conchitas, San Bartolo), Ica (Lagunillas, Playa Atenas), Arequipa (Mollendo).

El material fue colectado a mano, fijado en formol al 7\% y conservado en alcohol al 70\%. El estudio en el laboratorio permitió la determinación taxonómica de las especies consultando la bibliografía disponible, y para el ordenamiento sistemático se siguió a Millard (1997) con algunas modificaciones de Martins (1996). El material está depositado en el Museo de Historia Natural de la Universidad Nacional Mayor de San Marcos (MUSM) y en el Laboratorio de Biología y Sistemática de Invertebrados Marinos de la Universidad Nacional Mayor de San Marcos (LaBSIM).

\section{Sistemática}

\begin{tabular}{|c|c|}
\hline FAMILIA & $\begin{array}{l}\text { EllobiIdAe H \& A. AdAms IN } \\
\text { PfeIfFER, } 1854\end{array}$ \\
\hline SUBFAMILIA & $\begin{array}{l}\text { EllobiInAe H \&A. AdAmS IN } \\
\text { Pfeiffer, } 1854\end{array}$ \\
\hline & ELLOBIUM RÖDING, 1798 \\
\hline SubGENUS & Auriculodes STRAND, 1928 \\
\hline
\end{tabular}

Ellobium (A.) stagnale (Orbigny, 1835) (Figura 1)

Auricula stagnalis Orbigny, 1835 in Guerin: 23.

Ellobium stagnalis, Skoglund, 1992:129; Cruz y Jiménez, 1994:159, fig.054; Keen, 1971: 849, fig. 2416.

Ellobium (Auriculodes) stagnale; Martins,1996:286.

Concha alargada con abertura más amplia en la parte anterior; columela con dos pliegues y labio externo liso; escultura con estrías longitudinales y finas pústulas en hileras espi- rales, bajo la sutura de la vuelta corporal; coloración blanco cremosa. Longitud, 18,5 mm.

Distribución: El Salvador hasta Ecuador e Isla del Coco, Costa Rica (Cruzy Jiménez, 1994).

Nueva Localidad: Tumbes (Malpelo).

Hábitat : Varado.

Material examinado: 1 lote, 1 conchilla; Col. A. Indacochea, 03.89; MUSM.

Observaciones: La conchilla se encontró en la arena seca de una poza langostinera. Nuevo registro para la costa Peruana.

Genus $\quad$ SARNIA H \& A. AdAms , 1855

Sarnia frumentum Petit, 1842

(Figura 2)

Conovulus frumentum Petit, 1842: 32.

Melampus (Siona) frumentum; Dall, 1909:204;

Letelier, et al., 2003:94.

Sarnia frumentum; Keen, 1971:850, fig.2418; Alamo y Valdivieso, 1987:92; Marincovich, 1973:41;

Letelier et al; 2003:94.

Concha cilindroide con abertura alargada y espira corta; columela con tres pliegues, siendo el central más desarrollado; labio externo con el borde liso y engrosado interiormente; escultura con finas estrías longitudinales; coloración pardo claro con la espira blanquecina. Longitud, 7,4 mm.

Distribución: Callao, Perú a Chañaral, Chile (Marincovich, 1973).

Localidades: Lima (La Punta, Las Conchitas, San Bartolo); Ica (Lagunillas).

Hábitat: Playa de canto rodado en el Medio Litoral superior.

Material examinado: 20 lotes, 499 ejemplares (LaBSIM, MUSM).

Observaciones: Especie carroñera. Ha sido observada alimentándose de restos de aves, peces, y erizos.

Subfamilia Melampinae Stimpsom, 1851

GenUS MELAMPUSMONTFORD,1810

Subgenus Melampus S.S 


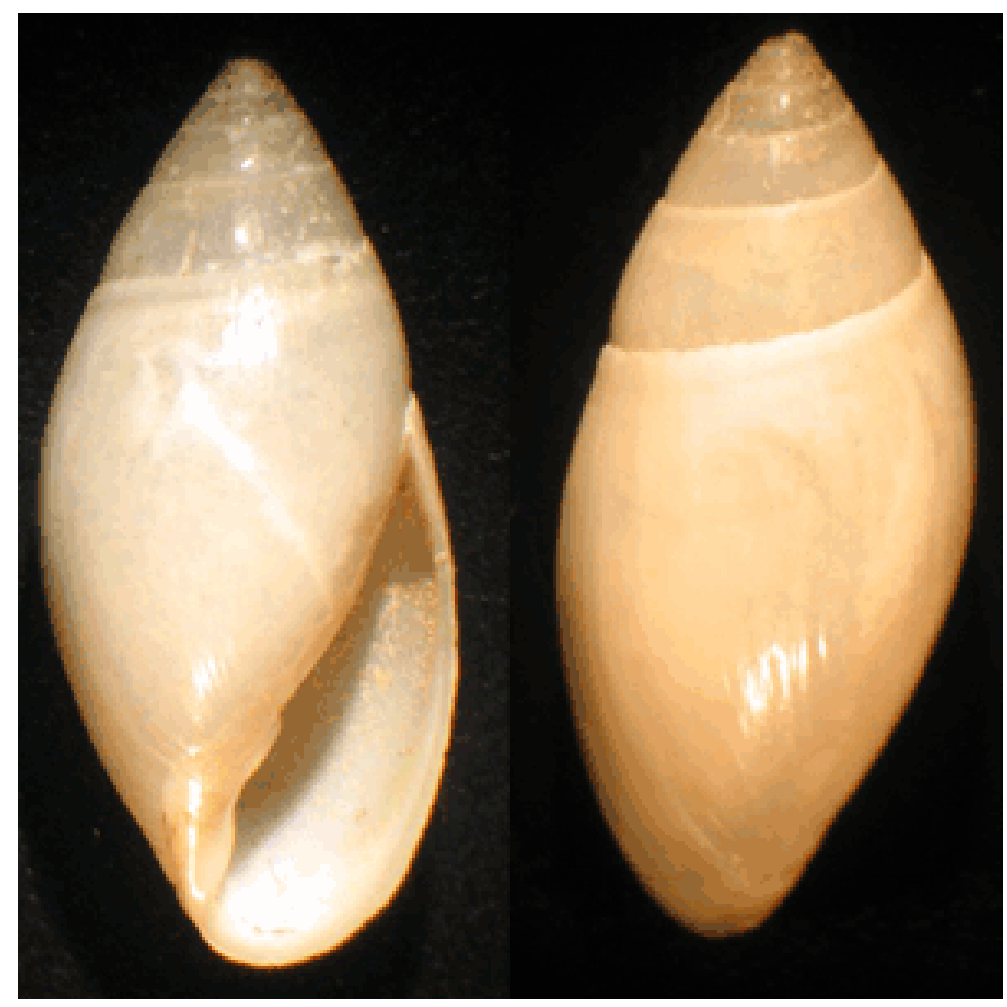

Figura 1. Ellobium (A.) stagnale, longitud $18,5 \mathrm{~mm}$.

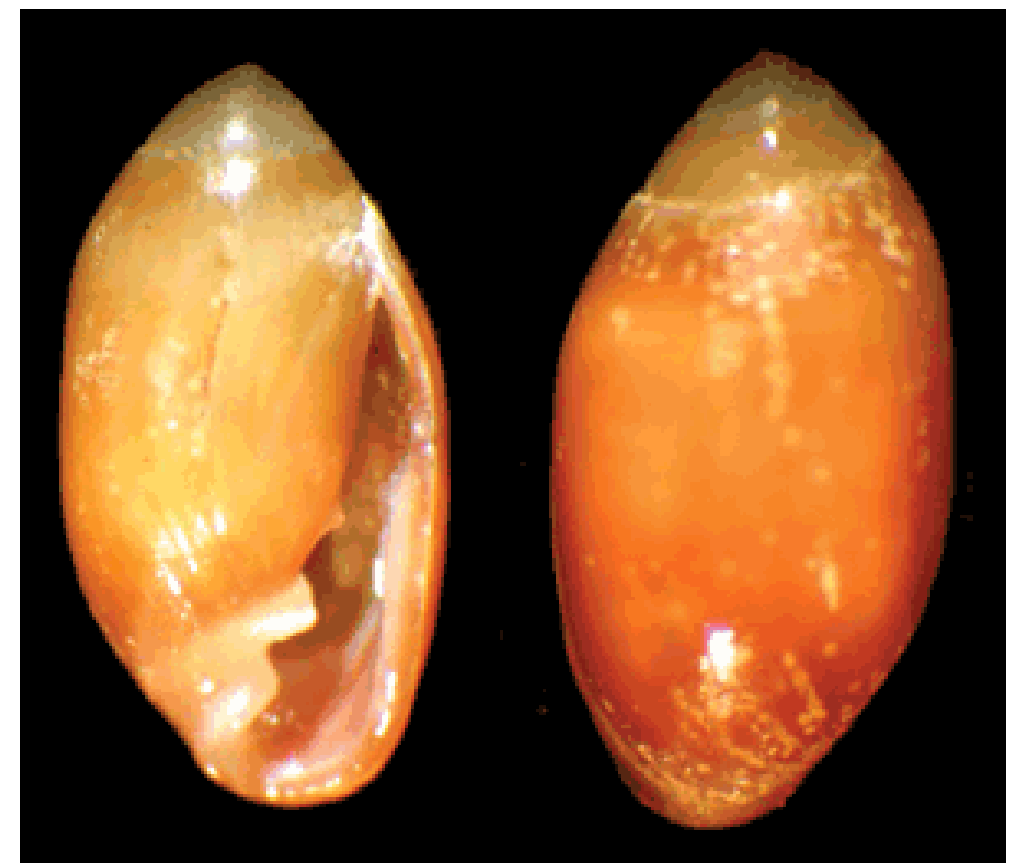

Figura 2. Sarnia frumentum, longitud $7,4 \mathrm{~mm}$ 


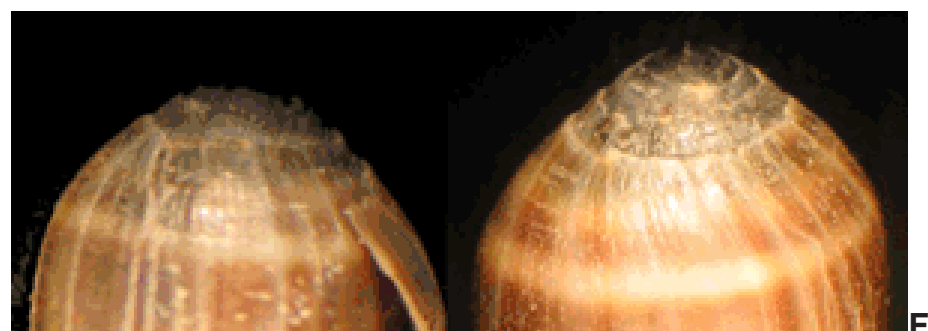

Figura 3. Melampus (M.) carolianus, longitud 13,2 mm

Figura 4. Melampus (M.) olivaceus, longitud $17,5 \mathrm{~mm}$.

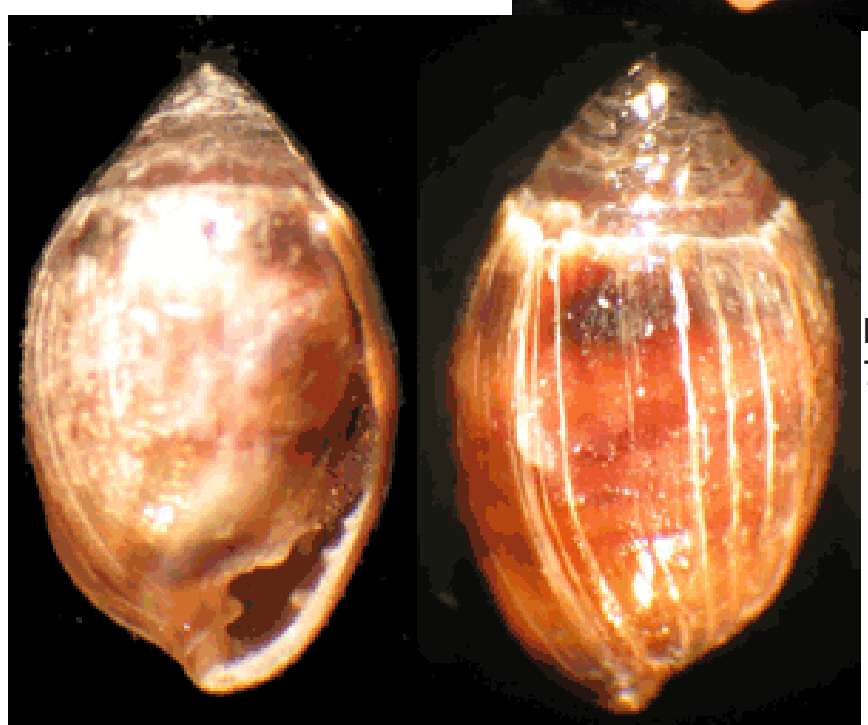

Figura 5. Detracia gramínea, longitud $7,5 \mathrm{~mm}$ 
Melampus (M.) carolianus (Lesson, 1842)

(Figura 3)

Auricula ambigua Lesson, 1842

Melampus piriformis, Dall, 1909:204

Melampus (Melampus) carolianus; Keen,1971:84 fig.2398; Alamo y Valdivieso, 1987:92, fig.188;

Skoglund, 1992:129; Cruz y Jiménez, 1994:154, fig.049.

Concha ovoide con espira corta y abertura alargada; numerosos pliegues columelares, siendo los posteriores débiles, y el interior del labio externo con fuertes liraciones; color gris parduzco con tres bandas espirales blanco amarillentas en la vuelta corporal. Longitud, 13,2 mm.

Distribución: Costa Rica hasta Tumbes, Perú; Islas Galápagos, Ecuador e Isla del Coco, Costa Rica (Cruz y Jiménez, 1994).

Localidades: Tumbes (E1 Bendito, Puerto Pizarro); Piura (Manglar de San Pedro).

Hábitat: Sobre la arena húmeda entre la vegetación herbácea del borde del manglar y bajo la hojarasca.

Material examinado: 5 lotes, 110 ejemplares (MUSM, LaBSIM).

Observaciones: Resultó la especie más abundante en los lugares de estudio, conjuntamente con $D$. graminea. Es el único elóbido que se presenta en el relicto de manglar de San Pedro, Piura (Peña, com. pers.).

\section{Melampus (M.) olivaceus Carpenter, 1857}

(Figura 4)

Melampus (Melampus) olivaceus Carpenter, 1857; McLean, 1969:59, fig. 33(2); Keen, 1971: 846, fig. 2400; Skoglung, 1992: 129; Goto y Poppe, 1996: 63.

Concha lisa ovoide, sólida, con espira baja y hombros redondeados; la abertura alargada sobrepasa el hombro; columela con un pliegue bajo anterior y un pliegue más desarrollado cerca del centro; labio externo con 12-13 cuerdas interiores cuyos extremos externos se elevan como dentículos; color pardo purpúreo claro con bandas espirales blanquecinas, la más notoria atravesando los hombros. Longitud, 17,5 mm.
Distribución: Lago Mugu, California a Mazatlán, México y Golfo de California (McLean, 1969); y Sur de Colombia (von Prahl, Cantera y Contreras, 1990).

Nueva localidad: Tumbes (Puerto Pizarro).

Hábitat: Fuera del manglar, en la línea de pleamar, entre la vegetación herbácea sobre la arena húmeda (McLean, 1969).

Material examinado: Un lote, 3 conchillas; Col. C. Paredes, 18.12.75; MUSM.

Observaciones: Las conchillas se encontraron varadas, entre la grama seca cerca del manglar junto con conchillas de M. carolianus. Nuevo registro para la costa Peruana.

Genus Detracia Gray in Turton,1840

Detracia graminea Morrison ,1946

(Figura 5)

Detracia graminea Morrison,1946; Keen, 1971:846, fig.2403; Cruz y Jiménez,1994:155, fig.050.

Concha pequeña ovoide con abertura alargada y tres pliegues columelares, dos posteriores pequeños y un gran pliegue anterior: labio externo con cuerdas finas en el interior; coloración marrón, con bandas espirales más oscuras. Longitud, 7,5 mm.

Distribución: Panamá hasta Guayaquil, Ecuador e Islas Perlas, Panamá (Cruz y Jiménez, 1994).

Nueva localidad: Tumbes (El Bendito, Puerto Pizarro, Isla del Amor).

Hábitat: Sobre la arena húmeda bajo la hojarasca y entre la vegetación herbácea del borde del manglar.

Material examinado: Un lote, 7 ejemplares; Col. A. Indacochea, 01.87; MUSM.- Un lote, 29 ejemplares; Col. A. Indacochea, 01.87; MUSM.- Un lote, 77 ejemplares; Col. A. Indacochea,08.87; LaBSIM.- Un lote, 13 ejemplares; Col. C. Paredes, 20.02.3; LaBSIM.- Un lote, 43 ejemplares; Col. C. Paredes y F. Cardoso, 28.09.04; MUSM.- Un lote, 7 ejemplares; Col. C. Paredes y F. Cardoso, 28.09.04; LaBSIM.- 
1 lote, 17 ejemplares; Col. C. Paredes y F. Cardoso,29.09.04; LaBSIM.

Observaciones: Se hallaron numerosos ejemplares compartiendo el hábitat con M. Carolianus. Nuevo registro para la costa peruana.

\section{Subfamilia Pedipedinae Fischer \& Croose, 1880}

Genus MarinUla KING \& BRODERIP, 1832

Marinula acuta (Orbigny, 1835)

(Figura 6)

Auricula acuta Orbigny, 1835 in Guerin, 1835: 23, № 2.

Marinula acuta, Dall, 1909: 205; Keen, 1971: 849,

fig. 2413; Cruz y Jiménez, 1994: 158, fig. 053;

Goto y Poppe, 1996: 629.

Concha ovalada algo alargada; columela con tres pliegues, el posterior tiene forma de diente, y callo columelar bien delimitado; labio externo delgado; escultura consiste en estrías axiales con desarrollo variable; coloración marrón con bandas axiales más oscuras en la vuelta corporal. Longitud, 7,0 $\mathrm{mm}$.

Distribución: Costa Rica a Guayaquil, Ecuador ( Keen, 1971).

Nueva localidad: El Bendito, Tumbes, Perú.

Hábitat: Bajo la hojarasca, en el borde del manglar, sobre arena gruesa húmeda.

Material examinado: 1 lote, 3 ejemplares; $\mathrm{Col}$. C. Paredes y F. Cardoso, 28.09.04; LaBSIM.

Observaciones: Nuevo registro para la costa Peruana. Comparte el hábitat con Melampus carolianus y Detracia graminea.

\section{Marinula concinna (C.B.Adams, 1852)}

(Figura 7)

\section{Auricula concinna C.B. Adams, 1852}

Marinula concinna; Keen, 1971:849, fig. 2414; Alamo y Valdivieso, 1987: 92; Skoglund, 1992: 129; Cruz y Jiménez, 1994: 157, fig. 052.

Concha ovoide con la vuelta corporal amplia y la espira pequeña; escultura con estrías axiales; columela con tres dientes blancos, siendo más desarrollado el diente posterior; labio externo liso de color marrón oscuro; color de la concha pardo oscuro, con una banda estrecha negruzca bajo la sutura. Longitud: 9,9 $\mathrm{mm}$.

Distribución: Costa Rica hasta Tumbes, Perú (Cruz y Jiménez, 1994).

Hábitat: Bajo la hojarasca en el borde del manglar (Keen, 1971).

Material examinado: Un lote, 5 ejemplares (MUSM).

Observaciones: El material fue colectado en 1961 por Mario Peña, en Puerto Pizarro. No se ha encontrado ejemplares de esta especie.

\section{Marinula pepita King,1831}

(Figura 8)

\section{Marinula pepita King, 1831:344; Dall, 1909 :205 \\ Keen ,1971:849; Letelier et al ; 2003:94}

Concha ovoide con vuelta corporal amplia y la espira pequeña; escultura con estrías axiales fuertes y un pliegue bajo la sutura; columela con tres dientes, siendo más desarrollado el diente posterior; labio externo liso, engrosado interiormente; coloración pardo rojiza, columela y diente de color blanco. Longitud, 7,7 mm.

Distribución: Guayaquil, a la Isla Chiloe, Chile (Dall,1909).

Localidades: Lima (La Punta, Las Conchitas, San Bartolo).

Hábitat: Playa de canto rodado en el Medio Litoral Superior.

Material examinado: 23 lotes, 957 ejemplares (MUSM, LaBSIM).

Observaciones: Keen (1971) reporta esta especie de Coquimbo a Isla Chiloe, y afirma que los reportes para Ecuador son erróneos. Ha sido observada alimentándose de restos de peces, Aves y erizos.

\section{Discusión y conclusiones}

Desde los estudios de Peña (1970, 1970a, 1970b), solamente Melampus carolianus y Marinula concinna han sido las especies de Ellobiidae conocidas tradicionalmente para los manglares del Departamento de Tumbes, aun- 
Figura 6. Marinula acuta, longitud 7,0 mm
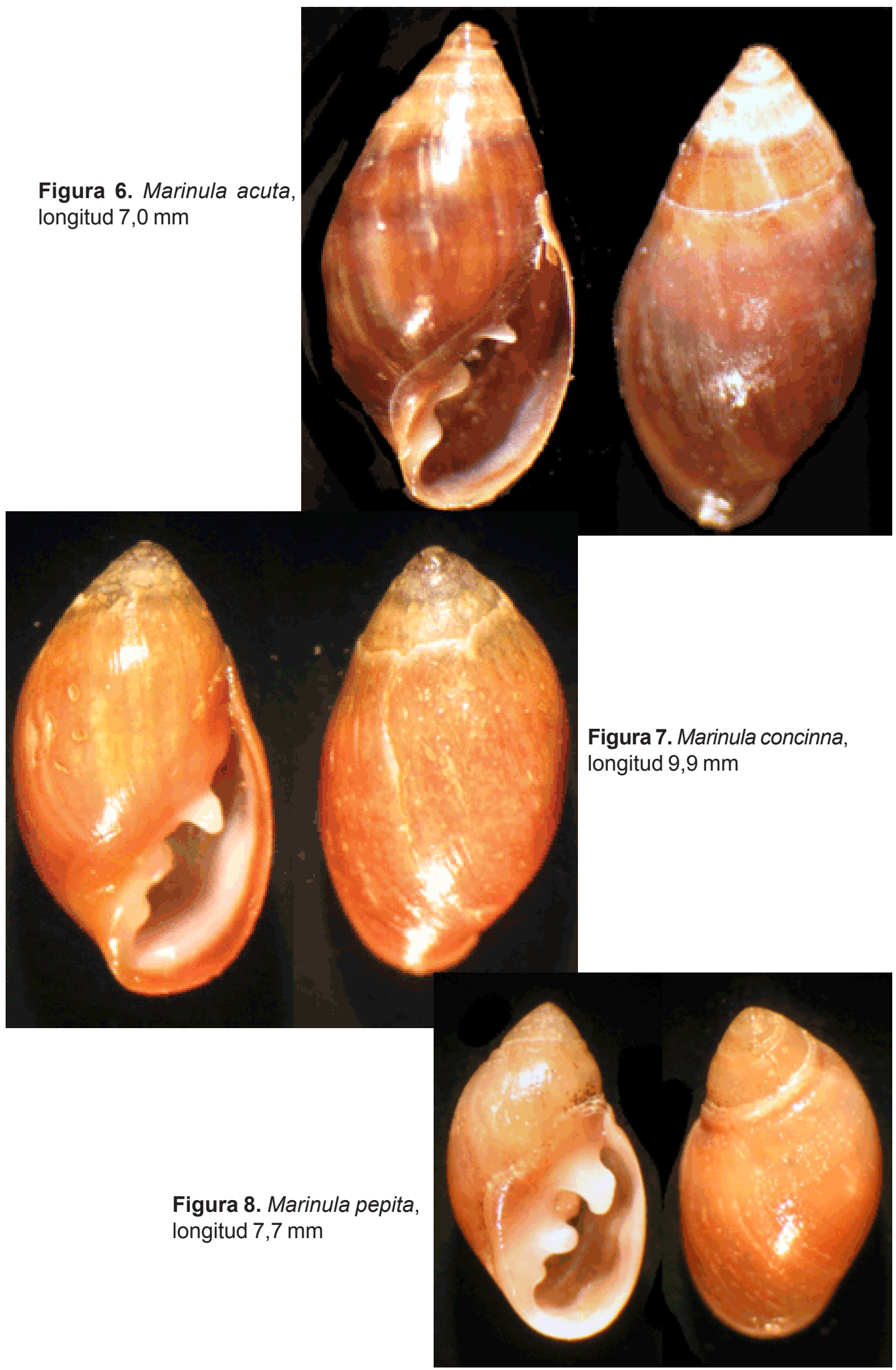
que desde entonces no ha sido hallada M.concinna.

Es posible que otros Ellobiidae vivan asociados a los manglares peruanos, puesto que en una sola colecta realizada en el poblado El Bendito, cerca de la frontera con Ecuador, en setiembre del año 2004, se encontró abundantes ejemplares de Melampus carolianus y Detracia gramínea; y tres ejemplares de Marinula acuta, bajo la hojarasca en el borde del manglar. Las dos últimas especies son nuevos registros, a los que hay que añadir Ellobium stagnale y Melampus olivaceus, colectados en años anteriores.

En el área de la Provincia Peruana son conocidos en el nivel supralitoral de las playas de canto rodado, Marinula pepita y Sarnia frumentum, especies comunes en la localidad de La Punta, Callao. Además, se ha colectado esporádicamente desde el año 1982 la especie Pedipes sp. aún no determinada. Igualmente, desde el año 1990 la especie Tralia sp. fue hallada en la playa pedregosa de la Bahía de Paracas, Ica, viviendo en la superficie inferior de las piedras aplanadas, del mediolitoral. Finalmente, en junio del año 2003, en la playa arenofangosa de La Arenilla, La Punta, Callao, localidad visitada por lo menos dos veces al año, durante unos 25 años, fueron colectados por única vez 13 ejemplares de un Ellobiidae, aún no determinado, el cual podría ser Tralia reflexilabris, reportada para el Callao por Dall (1909), quien cita también a Marinula marinella en la misma localidad.

En conclusión, con este estudio se incrementa de cuatro a ocho, el número de gasterópodos pulmonados de la familia Ellobiidae, reportados para la costa peruana; de las cuales, seis viven asociadas al manglar del Departamento de Tumbes, y dos habitan las playas de canto rodado en diversas localidades de la Provincia Peruana. Si se añaden las tres especies con determinación taxonómica pendiente, se incrementa a once el número de elóbidos hallados hasta el presente en el mar peruano.

\section{Literatura citada}

Alamo V.y V. Valdivieso. 1987. Lista sistemática de moluscos marinos del Perú. Bol. Inst. Mar. Perú- Callao. Volumen Extraordinario: 205 pp., 406 figs.

Carcelles A.R. y S.I. Williamson. 1951. Catálogo de los Moluscos Marinos de la Provincia Magallanica. Rev. Inst. Nac. de Inves. Cient. Nat. (Argentina). Cien. Zool. 2(5): 225-383.

Cruz R.A. y J.A. Jiménez. 1994. Moluscos asociados a las áreas de manglar de la Costa Pacífica de América Central: Guía. Heredia, C.R.: EFUNA. 182 pp.

Dall W.H. 1909. Report on a collection of shells from Perú, whith a summary of the littoral marine Mollusca of the Peruvian Zoological Province. Proc. U.S.N. Mus. 37(1704): 147-294.

Goto Y. \& G.T. Poppe. 1996. A listing of living Mollusca. Part II. Volume 1. Ed. L'Informatore Piceno, Ancona, Italy. 520 pp.

Hyman L.H. 1967. The Invertebrates: Volume VI, Mollusca I. McGraw-Hill Book Company. N.Y. 792 pp.

Keen A.M. 1971. Sea shells of tropical West America. 2a. ed. Stanford Univ. Press, California, 1064 pp., 3325 figs. 22 pls.

Letelier S., M.A. Vega, A.M. Ramos y E. Carreño. 2003. Base de datos del Museo Nacional de Historia Natural: moluscos de Chile. Rev. Biol. Trop. 51 (Suppl.3): 33-137.

McLean J.H. 1969. Marine Shells of Southern California. Science Series 24, Zoology 11: 104 pp.

Marincovich L. 1973. Intertidal Mollusks of Iquique, Chile. Los Angeles Co. Mus. Nat. Hist., Sc. Bull. 16:1-49, 102 figs.

Martins A.M.F. 1996. Relationships within the Ellobiidae. In: Origin and evolutionary radiation of the Mollusca (ed. J. Taylor), pp. 285-294, Oxford University Press.

Millard V. 1997. Classification of Mollusca: A classification of worldwide Mollusca. South Africa. 544 pp.

Paredes C., P.Huaman, F. Cardoso, R. Vivary V. VERA.1999. Estado actual del conocimiento de los moluscos acuáticos del Perú. Rev. peru. biol. 6(1) : 5-47.

Peña M. 1970. Zonas de distribución de los gasterópodos marinos el Perú. Anales Científicos Univ. Nac. Agraria. 8 (3/4): 153-170.

1970 a. Biocenosis de los manglares peruanos. Anales Científicos Univ. Nac. Agraria. 9 (1/2): 38-45. 1970 b. Descripción de los gasterópodos de los manglares del Perú. Anales Científicos Univ. Nac. Agraria. 9 (1/2): 46-55.

Skoglund C. 2002. Panamic Province Molluscan literature. Additions and Changes From 1971 through 2001. III Gastropoda. The Festivus 33(Suppl.): 1-286.

Vaught K.C. 1989. A Classification of the living Mollusca. Florida: American Malacologist Inc., 189 pp. 